\title{
Evaluation of the effect of Teucrium polium ointment on the episiotomy wound healing in primiparous women
}

\author{
Atena Mohammady Rouzbahani ${ }^{1 \oplus}$, Mahrokh Dolatian $^{2 *(}$, Tayebeh Jahedbozorgan $^{3}{ }^{\circledR}$, Faraz Mojab $^{4}$, Majid Tajik $^{5}$ \\ ${ }^{1}$ Student Research Committee, School of Nursing and Midwifery, Shahid Beheshti University of Medical Sciences, Tehran, Iran \\ ${ }^{2}$ Department of Midwifery and Reproductive Health, School of Nursing and Midwifery, Shahid Beheshti University of Medical Sciences, \\ Tehran, Iran \\ ${ }^{3}$ Department of Obstetrics and Gynecology, Shahid Beheshti University of Medical Sciences, Mahdieh Hospital ,Tehran, Iran \\ ${ }^{4}$ Pharmaceutical Sciences Research Center (PSRC), Shahid Beheshti University of Medical Sciences, Tehran, Iran \\ ${ }^{5}$ Shahid Beheshti University of Medical Sciences, Tehran, Iran
}

\section{*Correspondence to} Mahrokh Dolatian, Email: mhdolatian@gmail.com, m_dolatian@sbmu.ac.ir

Received 14 Dec. 2020 Accepted 10 Feb. 2021 Published online 1 Apr. 2021

Keywords: Teucrium polium, Primiparous, Wound healing

\begin{abstract}
Introduction: Episiotomy is a surgical incision at the vaginal opening performed in the second stage of labor during childbirth. The purpose of performing an episiotomy is to increase the size of the soft tissue of the pelvis floor and prevent damage to the perineum, facilitate delivery, and reduce the time during childbirth.

Objectives: This study aimed to determine the effect of Teucrium polium ointment on wound healing in primiparous women.

Patients and Methods: This triple-blinded clinical trial was conducted on 84 primiparous women referred to Mahdieh hospital in Tehran. Participants randomly received $2 \%$ T. polium ointment and placebo. The first time the ointment was started 24 hours after delivery and continued twice a day for 10 days. Wound healing rate was measured by redness, edema, ecchymosis, discharge, and approximation (REEDA) scale on days 1, 5, and 10 postpartum. The data were analyzed using SPSS version 23.

Results: The results were performed on 84 women, 43 people using T. polium ointment and 41 people using placebo ointment. There was no significant difference between the two groups regarding obstetrics and demographic characteristics at the beginning of the study. The mean scores of REEDA in the T. polium group in the first 12 hours, days 5 and 10 were significantly different from the placebo group $(P<0.001)$.

Conclusion: Wound healing following episiotomy can be accelerated due to antibacterial, anti-inflammatory, and antiseptic properties of $T$. polium ointment.

Trial Registration: The trial protocol was approved by the Iranian registry of clinical trial (\#IRCT20100130003226N18; https://en.irct.ir/trial/40004, ethical code\# IR.SBMU.RETECH.1398.055).
\end{abstract}

Citation: Mohammady Rouzbahani A, Dolatian $M$, Jahedbozorgan T, Mojab F, Tajik M. Evaluation of the effect of Teucrium polium ointment on the episiotomy wound healing in primiparous women. Immunopathol Persa. 2021;7(2):e33. DOI: 10.34172 ipp. 2021.33

\section{Introduction}

Episiotomy is a surgical incision at the vaginal opening performed in the second stage of labor during childbirth $(1,2)$. The purpose of performing an episiotomy is to increase the size of the soft tissue of the pelvis floor, prevent damage to the perineum, facilitate delivery, and reduce the time during childbirth. The prevalence of episiotomy is $92 \%$ in Romania, $100 \%$ in Vietnam, 64\% in the United Arab Emirates, 52.9\% in China, 85\% in Australia, and $69.1 \%$ in Nigeria (3-5). No comprehensive statistics on episiotomy have been published in Iran; however, episiotomy is a common practice in Iranian hospitals. Complications of episiotomy include perineal rupture, bleeding, edema and wound infection, sphincter

\section{Key point}

Our triple-blinded clinical trial on a group of primiparous women showed the antibacterial, antiinflammatory and antiseptic properties of $T$. polium ointment following episiotomy.

and anal mucosal injury, urethral injury, bladder injury, and hematoma formation. Additionally, perineal pain, vaginal purulent discharge, wound opening, fever, and bacteremia can emerge following wound infection $(6,7)$.

The pain of episiotomy site is a stressful factor for the mother that interferes with medical care and daily activities. It causes the mother to change her attitude, activities,

\footnotetext{
Copyright $(\subset 2021$ The Author(s); Published by Nickan Research Institute. This is an open-access article distributed under the terms of the Creative Commons Attribution License (http://creativecommons.org/licenses/by/4.0), which permits unrestricted use, distribution, and reproduction in any medium, provided the original work is properly cited.
} 
mental involvement, and motherly feelings toward the baby (8). Various efforts can reduce perineal pain including maintaining perineal hygiene, keeping the wound dry, and using a variety of treatments that are both pharmacological and non-pharmacological (9). According to the World Health Organization (WHO), 25\% of the common drugs are of plant origin, $74 \%$ of which are traditionally transmitted from the past due to their medicinal effects, and today $80 \%$ of the world's population use medicinal plants to treat their clinical problems (10). Many patients also show a strong tendency to use herbs due to the low side effects and negative effects of some chemical drugs (11). The increasing need for medicines, the compatibility of medicinal plants with the body, and the emphasis of WHO on replacing chemical medicines with natural medicines have led to an increase in prescription and consumption of medicinal plants. Additionally, the side effects of chemical drugs have increased the consumption of medicinal plants in health and pharmaceutical industries (12).

According to phytochemical studies, Teucrium polium L. (Lamiaceae) contains various compounds such as essential oil, tannins, sterols, terpenoids, flavonoids, iridoids, and phenolics (13).

Flavonoids are low-molecular weight compounds that have long been known for their anti-inflammatory, antioxidant, wound healing, anti-allergy, liver protective, anti-clotting, anti-viral, and anti-cancer effects. They are known to reduce vascular mortality and prevent cardiovascular disease (14). The effect of hydroalcoholic extract of T. polium on wound healing process is due to its phenolic and flavonoid compounds, which stimulates healing by enhancing fibroblast proliferation, collagen deposition and maturation, epithelial junction, angiogenesis, and infiltration of inflammatory cells (15).

\section{Objectives}

A review of previous studies has shown that no research has been conducted so far on the effect of T. polium on the healing of perineal ulcers. Therefore, considering the known anti-inflammatory and anti-ulcer effects of T. polium, this study was performed to determine the effect of teal ointment on episiotomy wound healing in primiparous women.

\section{Patients and Methods}

\section{Study design}

This triple-blind clinical trial study was conducted on 84 eligible primiparous women referring to Mahdieh hospital in Tehran from November 11, 2019 to February 11, 2020 for normal delivery; 43 people used $T$. polium ointment $2 \%$, and 41 people used placebo ointment. Both T. polium ointment and placebo (eucerin) made by FM. T. polium was purchased from an herbal market (Tehran). After identification and confirmation, the herb was extracted with ethanol (96\%) (maceration X3). Then, the ethanol was evaporated and the herb extract was mixed with eucerin (2\%) and filled into tubes. The plant was washed after identification and confirmation. The parts used were then dried separately on flat paper at $35^{\circ} \mathrm{C}$ for three days. The dried samples were pulverized using a mortar and an electric mixer. In order to extract the T. polium, a modified massage method was used in which the powder of each plant was mixed with organic solvents of methanol (96\%) and ethanol (80\%) with a mass-volume ratio of $1: 10$ and at a temperature of $40^{\circ} \mathrm{C}$, respectively, and stirred regularly for 18 to 24 hours in a shaker incubator of the Pharmacy Faculty of Shahid Beheshti University of Medical Sciences.

The ointments were autoclaved before use. Inclusion criteria were primiparous women aged 18-35 years, resident of Tehran province, able to write and read, singleton pregnancy with live fetus and cephalic display, gestational age between 32-37 weeks, infant weight between 2500 to 4000 grams, body mass index (BMI) 18.5 to $25 \mathrm{~kg} / \mathrm{m}^{2}$ at the beginning of pregnancy, vaginal delivery with medial lateral episiotomy without rupture, no underlying diseases, preeclampsia, eclampsia, gestational diabetes, placental abruption, chorioamnionitis, severe anemia in pregnancy, and diseases that interfere with wound healing, not taking any drugs that affect wound healing such as corticosteroids, anticoagulants, antiepileptic, and immunosuppressant, having no allergies or history of allergies to topical herbal medicines, and having a disorder in delivery process.

Exclusion criteria were unwillingness to continue to participate in the study, failure to visit on designated days, not using ointments regularly, having an allergy to T.polium ointment, the need for perineal repair after episiotomy, fever and puerperal infection, severe postpartum constipation during the study, and hemorrhoids or postpartum anal fissure.

The data were collected using demographic information form, obstetric and delivery characteristics, antibiotic registration form, drug side effects, and health status assessment. The health status form included eight questions in the field of health issues.

The objectives of the study were presented to eligible mothers, and then written consent was obtained. The participants randomly received $2 \% \mathrm{~T}$. polium ointment and placebo using Excel software. The first time the ointment started was $24 \mathrm{~h}$ after delivery and continued twice a day for 10 days. Wound healing rate was measured by redness, edema, ecchymosis, discharge, and approximation (REEDA) scale on days 1,5 , and 10 postpartum (16).

To blind the research, the drug and placebo were coded by the pharmacist and the researcher; the subjects and analyst did not know the content of the ointment. The type of episiotomy, the method of repair, the delivery agent, and the repairing person were similar. After the initial evaluation of the episiotomy wound, the first intervention was performed by the mother with the guidance of the researcher.

After washing the hands and washing and drying the perineal area, the mothers applied the ointment about the 
size of a fingertip on the episiotomy area on the sutured site, twice a day for 10 days after delivery. According to the hospital routine, $500 \mathrm{mg}$ of cephalexin capsules was administered to all samples every 6 hours for 7 days. The mothers went to the gynecology clinic of the same hospital on days 5 and 10 after delivery. The REEDA scale was used to assess perineal improvement in lithotomy (16). On the following days, the form of side effects of the drug (allergy, infection, severe pain, itching, burns, and dryness in the wound area, fever, and chills) was completed for each sample, and they were asked to contact the researcher in case of any of these side effects.

\section{Ethical issues}

The research was conducted in accordance with the tenets of the Declaration of Helsinki. The Ethics Committee of Shahid Beheshti University of Medical Sciences approved the study. The institutional ethical committee at Shahid Beheshti University of Medical Sciences accepted all study protocols (IR.SBMU.RETECH.1398.055). Accordingly, written informed consent was taken from all participants before any intervention. The trial protocol was approved in the Iranian registry of clinical trial (identifier: IRCT20100130003226N18; https://en.irct.ir/ trial/40004). This study was extracted from MSc thesis of Atena Mohammady Rouzbahani at this university (Thesis\#17511).

\section{Data analysis}

SPSS software version 21 was used for data analysis. Mann-Whitney U test was used to compare the wound size before the intervention, and the Friedman test was used to compare the wound healing rate after the intervention. $\mathrm{P}$-value less than 0.05 was considered significant.

\section{Results}

The study was performed on 86 women. Two women from the placebo group were excluded from the study due to the lack of referral, and the results were performed on 84 women, 43 people using T. polium ointment and 41 people using placebo ointment (Figure 1). There was no statistically significant difference between the T. polium group and placebo group regarding age, education status of women and their spouses, employment status of women, income status, gestational age, BMI, neonatal birth weight, duration of the first, second, and third stages of labor, and the number of suture (Table 1).

Table 2 shows that the two groups had a statistically significant difference regarding REEDA scale 24 hours after delivery and also 5 and 10 days after delivery, and the T. polium group had a better effect regarding recovery over time compared to the placebo.

\section{Discussion}

The results showed that the use of $T$. polium ointment accelerates the healing of episiotomy wounds. There was no statistically significant difference between the two groups regarding the initial appearance of the wound. Symptoms of episiotomy wound healing (redness, edema, ecchymosis, discharge, and approximation) in the intervention group on days 1,5 , and 10 were significantly different, and the women in the intervention group had more improvement

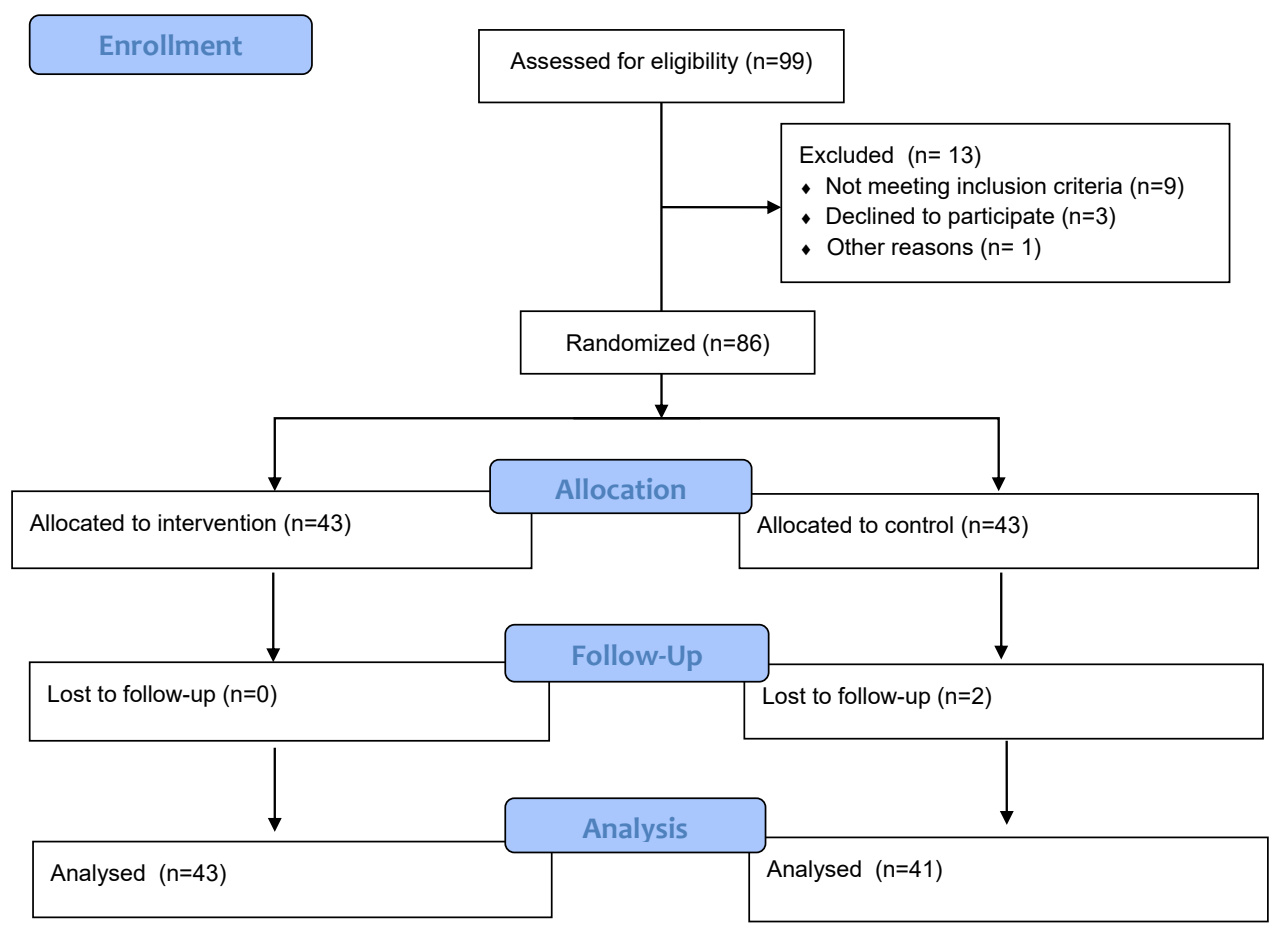

Figure 1. CONSORT; flow chart showing the flow of patients through the trial. 
Table 1. Comparison of demographic and obstetric characteristics of subjects in the Teucrium polium and placebo groups

\begin{tabular}{lccc}
\hline \multirow{2}{*}{ Variable } & Teucrium polium & Placebo & \multirow{2}{*}{ P value } \\
\cline { 2 - 3 } & Mean \pm SD & Mean \pm SD & \\
\hline Age $(\mathrm{y})$ & $24.85 \pm 3.77$ & $25.441 \pm 4.28$ & \\
\hline First stage of labor $(\mathrm{min})$ & $7.88 \pm 3.07$ & $8.27 \pm 3.08$ & 0.51 \\
\hline Second stage of labor $(\mathrm{min})$ & $42.34 \pm 14.75$ & $42.20 \pm 17.13$ & 0.55 \\
\hline Third stage of labor $(\mathrm{min})$ & $14.65 \pm 14.75$ & $13.93 \pm 5.40$ & 0.52 \\
\hline Gestational age $(\mathrm{wk})$ & $38.53 \pm 1.22$ & $38.69 \pm 1.22$ & 0.57 \\
\hline BMl $\left(\mathrm{kg} / \mathrm{m}^{2}\right)$ & $24.4 \pm 2.89$ & $24.39 \pm 3.07$ & 0.82 \\
\hline Number of suture & $5.46 \pm 0.50$ & $5.53 \pm 0.50$ & 0.80 \\
\hline Repair time $(\mathrm{d})$ & $24.79 \pm 8.59$ & $24.58 \pm 8.35$ & 0.905 \\
\hline
\end{tabular}

Table 2. Comparison of REEDA scale scores ( 12 hours, days 5 and 10 after delivery)

\begin{tabular}{lcccc}
\hline \multirow{2}{*}{ REEDA score } & $\mathbf{1 2}$ hours & $\mathbf{5}^{\text {th }}$ Day & $\mathbf{1 0}^{\text {th }}$ Day & \multirow{2}{*}{$\boldsymbol{P}$ value } \\
\cline { 2 - 4 } & Mean $\pm \mathbf{S D}$ & Mean $\pm \mathbf{S D}$ & Mean $\pm \mathbf{S D}$ & \\
\hline T. polium ointment & $0.74 \pm 0.44$ & $0.88 \pm 0.62$ & $0.51 \pm 0.88$ & $<0.001$ \\
Placebo ointment & $1.90 \pm 0.77$ & $1.73 \pm 0.70$ & $0.67 \pm 0.87$ & $<0.001$ \\
$P$ value & 0.0001 & 0.0001 & 0.005 & - \\
\hline
\end{tabular}

${ }^{\text {a }}$ Friedman test, ${ }^{\mathrm{b}}$ Mann-Whitney $U$ test.

than the control group; in other words, the improvement of episiotomy symptoms was observed over time. A review of the literature showed that studies on the effect of T. polium on wound healing in human samples are very limited; thereby, the results of this study were more consistent with studies performed in the laboratory settings.

In a randomized clinical trial study by Salih et al. on the effect of hydroalcoholic extract of T. polium on the healing process of diabetic wounds in rats, no statistically significant difference was observed between the two groups regarding initial wound appearance. There was a statistically significant difference regarding wound healing on days 7,14 , and 17 , and the intervention group subjects recovered more than the control group. Teucrium polium improves wound healing with a similar mechanism due to the presence of photonic compounds and flavonoids by increasing the rate of wound contraction, angiogenesis, and proliferation of fibroblasts collagen deposition, as is consistent with the results of the present study (15).

The anti-inflammatory substance containing flavonoids and sterols with antioxidant properties and immune components increased wound healing in the treatment group. In fact, phenolic compounds decrease the level of inflammatory factors that usually increase with wounds such as burning and thus increases the healing rate of subcutaneous wounds, which is consistent with the results of the present study (17).

Research has shown that flavonoids, as the main constituent of T. polium, have antioxidant properties and have a high ability to scavenge free radicals related to various diseases. Free radicals are found in many chronic health problems such as inflammation, and T. polium may suppress the oxidative process (18). Oxygen free radicals play an important role in wound healing at various stages of wound healing, but high levels of oxygen free radicals lead to oxidative stress, tissue cell death, and delayed wound healing (19); hence, antioxidants act to minimize damage (20). Therefore, the role of antioxidants in the wound healing process is important (21).

Since T. polium has antioxidant properties, this process is effective in wound healing. Another mechanism is the composition of ethanolic and methanolic extracts of the aerobic parts of the T. polium which has antibacterial properties and has been effective on both species of grampositive and gram-negative bacteria (22). Tannins are also the main constituents of T. polium that cause wound shrinkage, increase wound healing rate, and accelerate the healing of infectious wounds (23). No side effects were observed regarding the use of $T$. polium ointment.

\section{Conclusion}

According to the results, $T$. polium can be effective in healing episiotomy wounds. Therefore, T. polium can be considered as a wound healer. Further studies are recommended to be performed on the effectiveness of different doses and possible side effects.

\section{Limitations of the study}

Factors such as health and nutrition in the study individuals were impossible to be controlled; hence, we tried to control such problems by providing the same training and random selection of individuals. The strengths of the study include the control of intervening factors, face-to-face follow-up, and examination of the wound healing process by one person.

\section{Authors' contribution}

$A M R, M D$, and TJ were the principal investigators of the study. $M D, A M R$, and MT prepared the concept and design. MD and MT revised the manuscript and critically evaluated the intellectual contents. All authors participated in preparing the final draft of the manuscript, revised the manuscript, and critically evaluated the intellectual contents. All authors read and approved the content of the manuscript and confirmed the accuracy or integrity of any part of the work.

\section{Conflicts of interest}

The authors declare that they have no competing interests.

\section{Ethical considerations}

Ethical issues (plagiarism, data fabrication, and double publication) were completely observed by the authors.

\section{Funding/Support}

This study was funded by Shahid Beheshti University of Medical Sciences (Grant\#17511).

\section{References}

1. Jug Dosler A, Mivsek AP, Verdenik I. Incidence of episiotomy in Slovenia: The story behind the numbers. Nurs Health Sci. 
2017;19(3):351-357. doi:10.1111/nhs.12352

2. Hersh SR, Emeis CL. Mediolateral Episiotomy: Technique, Practice, and Training. J Midwif Womens Health. 2020;65:404409. doi: 10.1111/jmwh.13096.

3. Pasc A, Navolan D. A multicenter cross-sectional study of episiotomy practice in Romania. J Eval Clin Pract. 2019;25:306311. doi: 10.1111/jep.13062.

4. Trinh AT, Khambalia A, Ampt A, Morris JM, Roberts CL. Episiotomy rate in Vietnamese-born women in Australia: support for a change in obstetric practice in Viet Nam. Bull World Health Organ. 2013;91:350-6. doi: 10.2471/ BLT.12.114314.

5. Izuka E, Dim C, Chigbu C, Obiora-Izuka C. Prevalence and predictors of episiotomy among women at first birth in enugu, South-East Nigeria. Ann Med Health Sci Res. 2014;4:928-32. doi: 10.4103/2141-9248.144916.

6. Johnson A, Thakar R, Sultan AH. Obstetric perineal wound infection: is there underreporting? British J Nurs. 2012; $21: 28$ 30. doi: 10.12968/bjon.2012.21.Sup5.S28

7. Mittal M. Episiotomy. Labour Room Emergencies. Springer; 2020. p. 271-81

8. Mohamed H, El-Nagger NS. Effect of self perineal care instructions on episiotomy pain and wound healing of postpartum women. J Am Sci. 2012;8(6): 640-50.

9. Golezar S. Ananas comosus effect on perineal pain and wound healing after episiotomy: a randomized double-blind placebocontrolled clinical trial. Iran Red Crescent Med J. 2016 Mar 1;18(3):e21019. doi: 10.5812/ircmj.21019.

10. Ekor M. The growing use of herbal medicines: issues relating to adverse reactions and challenges in monitoring safety. Front Pharmacol. 2014 Jan 10; 4:177. doi: 10.3389/ fphar.2013.00177.

11. Rates SM. Plants as source of drugs. Toxicon. 2001;39(5):60313. doi: 10.1016/s0041-0101(00)00154-9.

12. Dubey N, Kumar R, Tripathi P. Global promotion of herbal medicine: India's opportunity. Current Sci. 2004; 86(1):37-41.

13. Elsonbaty S, Eltahawy N, Al-dmour S, Easa S, Qaralleh H. Ionizing radiation effect on Teucrium polium: phytochemical contents antioxidant and antibacterial activity. Arab J Nucl Sci Appl. 2020;53:98-110. doi: 10.21608/ajnsa.2020.15218.1242
14. Clericuzio M, Tinello S, Burlando B, Ranzato E, Martinotti S, Cornara L, et al. Flavonoid oligoglycosides from Ophioglossum vulgatum L. having wound healing properties. J Nat Med. 2012;78:1639-44. doi: 10.1007/s11418-013-0759-y.

15. Salih MA, Abdullah AN, Aziz S. Effect of hydroalcoholic extract of Teucrium polium on the process of wound healing in diabetic rats. Passer J. 2020;2(1):37-45. doi: 10.24271/psr.09

16. Alvarenga MB, Francisco AA, de Oliveira SM, da Silva FM, Shimoda GT, Damiani LP. Episiotomy healing assessment: Redness, oedema, ecchymosis, discharge, approximation (REEDA) scale reliability. Rev Lat Am Enfermagem. 2015;23:162-8. doi: 10.1590/0104-1169.3633.2538.

17. Sevindik E, Abacı ZT, Yamaner C, Ayvaz M. Determination of the chemical composition and antimicrobial activity of the essential oils of Teucrium polium and Achillea millefolium grown under North Anatolian ecological conditions. Biotechnol Biotechnol Equip. 2016;30:375-80. doi: 10.1080/13102818.2015.1131626

18. Shtukmaster S, Ljubuncic P, Bomzon A. The effect of an aqueous extract of Teucrium polium on glutathione homeostasis in vitro: a possible mechanism of its hepatoprotectant action. Adv Pharmacol Sci. 2010;2010:938324. doi: 10.1155/2010/938324.

19. Kurahashi T, Fujii J. Roles of Antioxidative Enzymes in Wound Healing. J Dev Biol. 2015:57-70. doi:10.3390/jdb3020057

20. Havsteen $\mathrm{BH}$. The biochemistry and medical significance of the flavonoids. Pharmacol Ther. 2002;96:67-202. doi: 10.1016/s0163-7258(02)00298-x

21. Süntar I, Nahar DL, Sarker S. Wound healing and antioxidant properties: Do they coexist in plants? Free Radic Biol Med. 2012;2:1-7. doi: 10.5530/ax.2012.2.2.122.

22. Autore G, Capasso F, De Fusco R, Fasulo MP, Lembo M, Mascolo N, Menghini A. Antipyretic and antibacterial actions of Teucrium polium (L.). Pharmacol Res Commun. 1984;16:219. doi: 10.1016/s0031-6989(84)80101-0.

23. Su X, Liu X, Wang S, Li B, Pan T, Liu D, et al. Wound-healing promoting effect of total tannins from Entada phaseoloides (L.) Merr. in rats. Burns. 2017;43:830-8. doi: 10.1016/j. burns.2016.10.010. 